Portland State University

PDXScholar

\title{
Molecular lifetimes in the presence of periodically roughened metallic surfaces
}

P.T. Leung

Portland State University

Z. C. Wu

Daniel A. Jelski

Thomas F. George

Follow this and additional works at: https://pdxscholar.library.pdx.edu/phy_fac

Part of the Physics Commons

Let us know how access to this document benefits you.

\section{Citation Details}

Leung, P. T., Wu, Z. C., Jelski, D. A., \& George, T. F. (1987). Molecular lifetimes in the presence of periodically roughened metallic surfaces. Physical Review B (Condensed Matter), 36(3), 1475-1479.

This Article is brought to you for free and open access. It has been accepted for inclusion in Physics Faculty Publications and Presentations by an authorized administrator of PDXScholar. Please contact us if we can make this document more accessible: pdxscholar@pdx.edu. 


\title{
Molecular lifetimes in the presence of periodically roughened metallic surfaces
}

\author{
P. T. Leung, Z. C. Wu, Daniel A. Jelski, and Thomas F. George \\ Departments of Physics and Astronomy and Chemistry, 239 Fronczak Hall, State University of New York at Buffalo, \\ Buffalo, New York 14260 \\ (Received 29 January 1987)
}

\begin{abstract}
The lifetimes of molecules located close to a sinusoidal grating surface are studied within a classical phenomenological model. The contribution of surface roughness to the molecular decay rate is attributed to the discrepancy between the experiments of Rossetti and Brus and the theory of Chance, Prock, and Silbey. It is found that surface roughness can either enhance or diminish the flat-surface value for the decay rate depending on the emitting frequency, molecule-surface distance, and the molecular orientation.
\end{abstract}

\section{INTRODUCTION}

The scattering of light and other fluorescence properties of molecules residing in the vicinity of a metallic surface have aroused immense interest since the early $1970 \mathrm{~s}^{1}$ especially after the discovery of the dramatic surface enhancement of Raman scattering (SERS). ${ }^{2}$ It has now become clear that in spite of many other possible effects, ${ }^{3}$ the roughness of the surface definitely plays an important role in leading to such dramatic surface phenomena. While in most experiments surface roughness unavoidably exists, the theoretical modeling of such roughness can generally be classified according to two categories: (1) random and (2) regular (periodic) roughness. A Gaussian distribution of roughness often serves as a prototype of the former, ${ }^{4}$ and the shallow sinusoidal grating surface is the simplest and typical example of the later. ${ }^{5}$ Other models, including a collective of bumps (represented by spheroids, either randomly or periodically distributed) have also been considered in the literature. ${ }^{6}$ In general, two distinct classes of phenomena are of particular interest as far as the role of the surface roughness is concerned: (1) the modification of the emission intensity and (2) the fluorescence lifetime of the admolecule in the presence of a rough metallic surface. One simple approach to such problems based on classical phenomenology $(\mathrm{CP})$ is to solve the Maxwell equations (ME) subject to these rough boundary conditions. The drawbacks of such an approach have been pointed out ${ }^{7}$ with possible remedies from a microscopic modeling of the surface electrons and the application of a nonlocal classical electromagnetic theory. ${ }^{8}$ It has been found that a CP approach is valid in general down to very small molecule-surface distances of a few angstroms. ${ }^{9}$ In particular, the problems of both the molecular dipole emission intensity ${ }^{10}$ and lifetime ${ }^{7}$ have been investigated by this approach, where the general solutions to the ME established by Maradudin and Mills ${ }^{11}$ and to the image problem by Rahman and Maradudin ${ }^{12}$ have been applied to surfaces with random roughness described by a Gaussian correlation function as

$$
\left\langle\xi\left(\mathbf{r}_{\|}\right) \xi\left(\mathbf{r}_{\|}^{\prime}\right)\right\rangle=\delta^{2} \exp \left(\left|\mathbf{r}_{\|}-\mathbf{r}_{\|}^{\prime}\right|^{2} / a^{2}\right)
$$

Here $\xi\left(\mathbf{r}_{\|}\right)$is the surface height at $\mathbf{r}_{\|}$, a position vector parallel to the $x y$ plane, and $\delta^{2}$ and $a$ are the mean-square height of the surface and the correlation length, respectively. We have taken $\hat{\mathbf{z}}$ to be the direction normal to the surface. For the case of periodic roughness, a shallow sinusoidal grating described by the correlation function

$$
\left\langle\xi\left(\mathbf{r}_{\|}\right) \xi\left(\mathbf{r}_{\|}^{\prime}\right)\right\rangle=\delta^{2} \sin (Q x) \sin \left(Q x^{\prime}\right),
$$

where $\delta$ is the grating amplitude and $Q$, the spatial grating period, has also been considered, and here the emission intensity has been found to exhibit pronounced angular resonances. ${ }^{13}$ One of the goals of the present paper is to complete this picture via a thorough study of the fluorescence lifetime of molecules near such grating surfaces. However, there are also other motivations leading to this study as described below.

On the experimental side, Kuhn ${ }^{1}$ and Drexhage ${ }^{14}$ are among the earliest investigators who employed the Langmuir-Blodgett ${ }^{15}$ fatty acid monolayer assembly technique to study the lifetime of various dye molecules separated by fixed distances from the metal layer evaporated onto glass substrates. Their results for molecules located not too close to the surface were described very well by the classical electromagnetic energy-transfer theory established by Chance, Prock, and Silbey (CPS) based on Sommerfeld's antenna theory. ${ }^{16}$ However, there are serious limitations to the fatty acid monolayer technique, ${ }^{15}$ as pointed out recently by Rossetti and Brus (RB), ${ }^{17}$ that only certain oxidized surfaces and lowfrequency emitting dyes can be studied by these experiments. By proposing a new "three-layer sandwich matrix" arrangement using argon as the spacer, RB studied the decay rates of pyrazine molecules near a silver surface and observed large discrepancies between the experimental data and the CPS theory at molecule-surface distances as large as $125 \AA$, with the theory predicting larger and ever increasing values and the experimental data showing a saturation tendency with values below the theoretical ones. This is a great surprise since many other similar experiments ${ }^{18}$ have shown that the classical theory is able to explain experimental results for molecule-surface distances down to a few angstroms. One possible way to 
save the classical theory and to explain the RB experiment is to take into account the effects of the surface roughness. After all, the CPS theory is good only for perfectly flat surfaces, and the perfect flatness of the silver boundary can hardly be guaranteed under these experimental conditions. ${ }^{17}$ Arias, Aravind, and Metiu ${ }^{7}$ have therefore studied theoretically the effects of surface roughness (assumed to be randomly distributed) on the molecular decay rate and have concluded such effects are irrelevant to RB's results, since the roughness has been found to add to the flat-surface value for the decay rate, thus bringing the CPS theory further away from the RB data. Moreover, it has been claimed that one can argue in more general terms that roughness always enhances the flat-surface values for the decay rate. ${ }^{9,19}$ However, the situation has since changed dramatically with the publication of a later paper ${ }^{20}$ by RB on similar experiments acknowledging that the earlier discrepancies found by them ${ }^{17}$ are erroneous. With modified experimental techniques, they no longer observed the previous saturation characteristics for the decay rates at small molecule-surface distances. Instead, the CPS theory is now found to agree very well with the experiment for a gold surface and to lie below the data for a silver surface. While RB have then attributed such discrepancies to the different reliability of the experimental values which they used for the dielectric constants for the metals, ${ }^{21}$ this later RB experiment surely revives the possibility that roughness may indeed play an important role in the comparison between theory and experiment. Furthermore, it was reported in the first RB paper that surface structure was observed via scanning electron microscope (SEM), and some sort of periodic structure seemed to exist (at least within a local region and to the limit of the resolving power of the SEM) on account of the surface finish of the underlying sapphire due to the polishing. ${ }^{17}$ This further motivates the present theoretical study. Besides the case of periodic roughness can provide a more quantitative comparison between theory and experiment since the degree of roughness can be controlled by various plating and grooving procedures.

In the following section, we shall present a systematic study of the molecular lifetimes on a shallow sinusoidal grating based on the image theory worked out by Rahman and Maradudin (RM). ${ }^{12}$ While being aware that this theory is not as accurate as the energy-transfer theory, ${ }^{16}$ it seems that it is not that straightforward to extend the Sommefeld antenna theory ${ }^{22}$ to the case of rough surfaces. With this left as an open problem, we resort back to the image theory and limit ourselves to situations where the molecule-surface distances are small compared to the emission wavelengths, in which case the two theories agree well with each other. ${ }^{16}$ In particular, we shall be interested in seeing the variation of the decay rates with emission frequency and molecule-surface distance for all three distinct orientations $(\hat{\mathbf{x}}, \hat{\mathbf{y}}, \hat{\mathbf{z}})$ of the molecular dipole. This latter feature is unique to the grating system, since in the other cases such as the flat or rough (with Gaussian randomness) cases, only two (parallel and perpendicular) distinct orientations are possible. One of the most interesting findings in this present study is that, depending on the orientation of the molecule and other factors, the effect of the roughness does not necessarily enhance the flat-surface values for the decay rate, in contrast to the anticipations of some previous investigators. ${ }^{9,19}$ Numerical results will be presented in Sec. III, after which, in Sec. IV, we shall discuss possible implications relevant to the experiments mentioned above.

\section{THEORY}

Similar to previous investigations, ${ }^{7}$ the present theory is based on the damped harmonic oscillator model ${ }^{16}$ for the molecule and the image theory for rough boundaries. ${ }^{12}$ The surface-induced decay rate obtained in this model can be expressed in terms of that for a free molecule (i.e., in the absence of the surface). For convenience of presentation, let us define two ratios of decay rates

$$
\begin{aligned}
R_{1} & \equiv \frac{\gamma_{R}}{\gamma_{0}}, \\
R_{2} & \equiv \frac{\gamma_{R}}{\gamma_{F}},
\end{aligned}
$$

where the subscripts $R, F$, and 0 stand for cases with a rough surface, flat surface, and a free molecule, respectively. Within this model, these ratios are found to be $\mathrm{e}^{7,16}$

$$
\begin{aligned}
& R_{1}=1+\frac{3}{2} \frac{q}{k^{3}} \operatorname{Im}\left(G^{F}+G^{R}\right), \\
& R_{2}=1+\frac{\frac{3}{2} \frac{q}{k^{3}} \operatorname{Im} G^{R}}{1+\frac{3}{2} \frac{q}{k^{3}} \operatorname{Im} G^{F}},
\end{aligned}
$$

where $q$ is the quantum yield of the emitting state, $k$ is the emission wave number, and the $\boldsymbol{G}(\omega)$ functions are defined as the image field on the molecular dipole per unit dipole moment, as a function of the emission frequency $\omega$, with $F$ and $R$ representing contributions from the flat boundary and from the roughness, respectively. To calculate these functions, we resort to the image theory of RM. $^{12}$ The image potential at a point $\mathbf{r}$ due to a point charge lying above the substrate surface at $\mathbf{r}^{\prime}$ [i.e., $\left.z^{\prime}>\xi\left(\mathbf{r}_{\|}^{\prime}\right)\right]$ is obtained as a solution of the Poisson equation and is given by [for $z>\xi\left(\mathbf{r}_{\|}\right)$]

$$
\phi\left(\mathbf{r} \mid \mathbf{r}^{\prime}\right)=\int \frac{d^{2} k_{\|}}{(2 \pi)^{2}} A\left(\mathbf{k}_{\|}\right) \exp \left(i \mathbf{k}_{\|} \cdot \mathbf{r}_{\|}\right) \exp \left(-k_{\|} z\right)
$$

where $\mathbf{k}_{\|}$is the two-dimensional wave vector on the $x y$ plane and the coefficient $\boldsymbol{A}\left(\mathbf{k}_{\|}\right)$is to be determined from matching appropriate boundary conditions. Following $\mathrm{RM},{ }^{12}$ we obtain the perturbative solution for $A\left(\mathbf{k}_{\|}\right)$for a sinusoidal boundary $\xi\left(\mathbf{r}_{\|}\right)=\delta \sin (Q x)$. To first order in the roughness $(\delta Q)$, we get $^{12,23}$

$$
A\left(\mathbf{k}_{\|}\right) \approx A^{(0)}\left(\mathbf{k}_{\|}\right)+A^{(1)}\left(\mathbf{k}_{\|}\right)
$$

where

$$
A^{(0)}\left(\mathbf{k}_{\|}\right)=-\frac{2 \pi e}{k_{\|}} \frac{\epsilon-1}{\epsilon+1} \exp \left(-i \mathbf{k}_{\|} \cdot \mathbf{r}_{\|}^{\prime}\right) \exp \left(-k_{\|} z^{\prime}\right),
$$

and 


$$
\begin{aligned}
A^{(1)}\left(\mathbf{k}_{\|}\right)=- & \frac{4 \pi e}{k_{\|} \widetilde{k}_{\|} Q} \frac{\epsilon-1}{(\epsilon+1)^{2}}(\delta Q)\left[\epsilon k_{\|} \widetilde{k}_{\|}+\mathbf{k}_{\|} \cdot\left(\mathbf{k}_{\|}-Q\right)\right] \\
& \times \exp \left[-i\left(\mathbf{k}_{\|}-Q\right) \cdot \mathbf{r}_{\|}^{\prime}\right] \exp \left(-\widetilde{k}_{\|} z^{\prime}\right)
\end{aligned}
$$

where

$$
\mathbf{Q} \equiv Q \widehat{\mathbf{e}}_{x}, \quad \tilde{k}_{\|}^{2} \equiv\left(k_{x}-Q\right)^{2}+k_{y}^{2},
$$

and $\epsilon$ is the complex dielectric constant of the surface substrate.

Substituting Eqs. (7)-(10) into (6), we get the image potential to first order in roughness. It is then straightforward to show the $A^{0}\left(\mathbf{k}_{\|}\right)$gives back the ordinary image potential for a flat boundary and $A^{(1)}\left(\mathbf{k}_{\|}\right)$yields the lowest-order effect of the roughness on $\phi\left(\mathbf{r} \mid \mathbf{r}^{\prime}\right)$. To calculate the $G$ functions as defined above, we must calculate from Eq. (6) the image electric field due to a dipole $\mu$ (at r) on the dipole itself. Hence, we have

$$
\mathbf{E}(\mathbf{r})=-\frac{1}{e}\left\{\nabla_{\mathbf{r}}\left[\boldsymbol{\mu} \cdot \nabla_{\mathbf{r}^{\prime}} \phi\left(\mathbf{r} \mid \mathbf{r}^{\prime}\right)\right]\right\}_{\mathbf{r}^{\prime}=\mathbf{r}} .
$$

Substituting Eqs. (6)-(10) into (11), we obtain the $G$ functions for a dipole $\boldsymbol{\mu}$ located at $(0,0, d)$ as indicated below.

(i) For the case of a perpendicular dipole

$$
\begin{aligned}
& G_{z}^{F}= \frac{\epsilon-1}{\epsilon+1} \frac{1}{4 d^{3}}, \\
& G_{z}^{R}=\frac{4 \delta}{\pi} \frac{\epsilon-1}{(\epsilon+1)^{2}} \int_{0}^{\infty} d u \int_{0}^{\infty} d v(\epsilon f g+h) \\
& \times \exp [-(f+g) d] .
\end{aligned}
$$

(ii) For the case of a parallel dipole

$$
\begin{array}{r}
G_{x}^{F}=G_{y}^{F}=\frac{\epsilon-1}{\epsilon+1} \frac{1}{8 d^{3}}, \\
G_{x}^{R}=\frac{4 \delta}{\pi} \frac{\epsilon-1}{(\epsilon+1)^{2}} \int_{0}^{\infty} d u \int_{0}^{\infty} d v \frac{u^{2}-\frac{Q^{2}}{4}}{f g}(\epsilon f g+h) \\
\quad \times \exp [-(f+g) d], \\
G_{y}^{R}=\frac{4 \delta}{\pi} \frac{\epsilon-1}{(\epsilon+1)^{2}} \int_{0}^{\infty} d u \int_{0}^{\infty} d v \frac{v^{2}}{f g}(\epsilon f g+h) \\
\times \exp [-(f+g) d],
\end{array}
$$

where $f, g, h$ are functions of $u, v$ given by

$$
\begin{aligned}
& f(u, v)=\left[\left[u+\frac{Q}{2}\right]^{2}+v^{2}\right]^{1 / 2}, \\
& g(u, v)=\left[\left[u-\frac{Q}{2}\right]^{2}+v^{2}\right]^{1 / 2}, \\
& h(u, v)=u^{2}+v^{2}-\frac{Q^{2}}{4} .
\end{aligned}
$$

Using the results from Eqs. (12)-(19) and assuming a quantum yield of approximate unity, ${ }^{16}$ we can compare the different decay rates [Eq. (3)] by performing numerical analysis on Eqs. (4) and (5), whose results are presented in the next section.

\section{NUMERICAL RESULTS}

For most of the calculations, we have taken the substrate as a silver sinusoidal grating surface with $\delta=0.7 \AA$ and $Q=0.08 \AA^{-1}$ (i.e., a roughness with $\delta Q \approx 0.05$ ), and the values for $\epsilon$ are taken from Ref. 21. As expected from the $G$ functions in Eqs. (13), (15), and (16), the contributions here are linear in the roughness and should therefore be an order of magnitude greater than those for random roughness. $^{7}$ Indeed, for a molecular-surface distance $d=20 \AA$, Fig. 1 shows a dramatic enhancement of the free-decay value $\left(\gamma_{0}\right)$ by means of a plot of $R_{1}$ versus the emitting frequency $\omega$. In particular, we find that under these conditions the perpendicular dipoles are more heavily damped than the parallel dipoles, while all of them show clearly the effect of the surface plasmon (SP) resonance at $\omega_{\mathrm{SP}} \cong 3.63 \mathrm{eV} .^{7,24}$ Figure 2 shows clearly the effect of the roughness by means of a plot of $R_{2}$ versus $\omega$ with the same parameters as in Fig. 1. We observe that with the present small value for $\delta Q$, the overall contribution of the roughness to the flat surface value is less than $20 \%$. While the SP resonances are exhibited as before, we notice that in the neighborhood of the SP resonance, the two parallel directions (with $\hat{\mathbf{x}}$ along the grating and $\hat{\mathbf{y}}$ along the grooves) show a kind of " $180^{\circ}$ out of phase" behavior with respect to each other. More interestingly, we observe that for some emitting frequencies, roughness does not necessarily lead to a larger value for the decay rate. Depending on $\omega, d$, and the molecular orientations, it is clear from the graphs that $R_{2}$ can dip below unity for certain values of $\omega$. This is contrary to the claim of some previous investigations ${ }^{9,19}$ and shall be further manifested

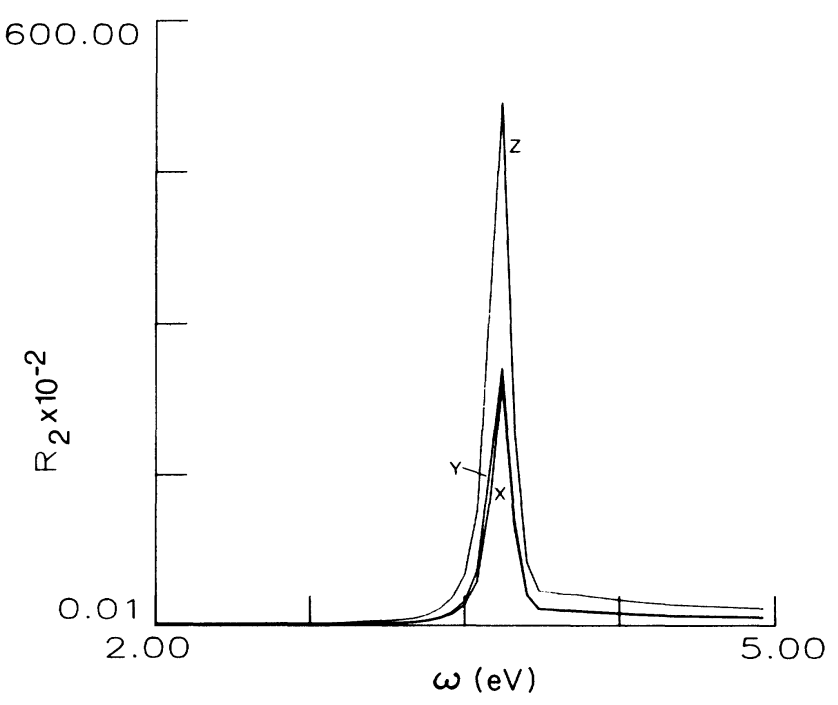

FIG. 1. Comparison of the molecular decay rates at a silver grating surface with that of a free molecular $\left(R_{1}\right)$ for different emitting frequencies $(\omega)$ and molecular orientations at $d=20 \AA$. The unit for $\omega$ is in $\mathrm{eV}$ for all figures, and other parameters are given in the text. 


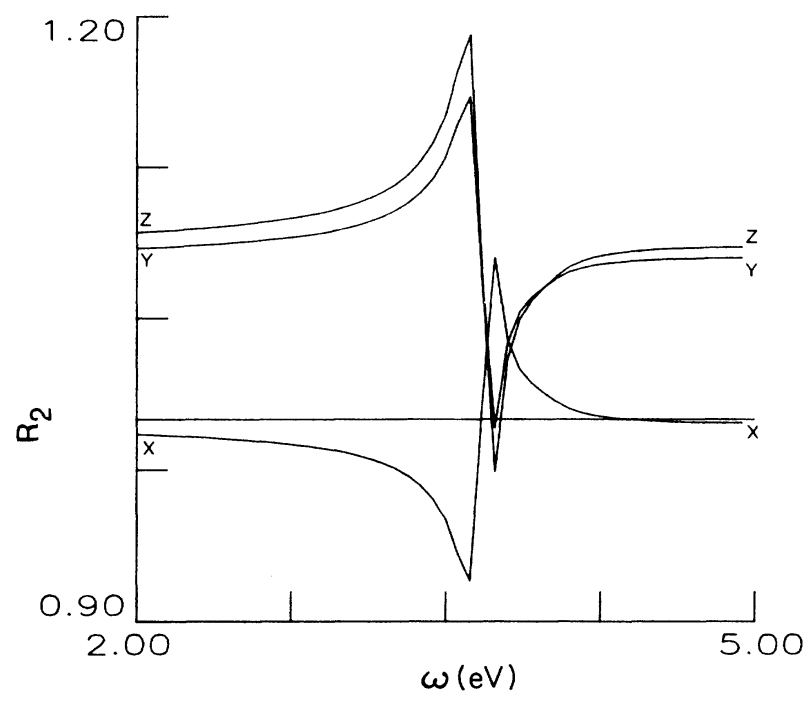

FIG. 2. Comparison of the molecular decay rates at the grating surface with that at a flat surface $\left(R_{2}\right)$ for different emitting frequencies and molecular orientations. All parameters are the same as in Fig. 1.

in the following figures. Figure 3 shows the variation of $R_{2}$ with $d$ at $\omega=3.74 \mathrm{eV}$, close to the SP resonance frequency $\omega_{\text {SP. }}$. We find that the $\widehat{\mathbf{x}}$ dipoles oriented along the grating are damped most heavily except at very close distances from the surface, whereas for the $\hat{\mathbf{y}}$ - and $\hat{\mathbf{z}}$-oriented molecules, it is clearly seen that the roughness again plays a role in diminishing further the decay rate for molecules located not too close $(d \geq 20 \AA)$ to the surface. However, the situation changes dramatically if one goes away from the SP resonance. Figure 4 shows similar results as in

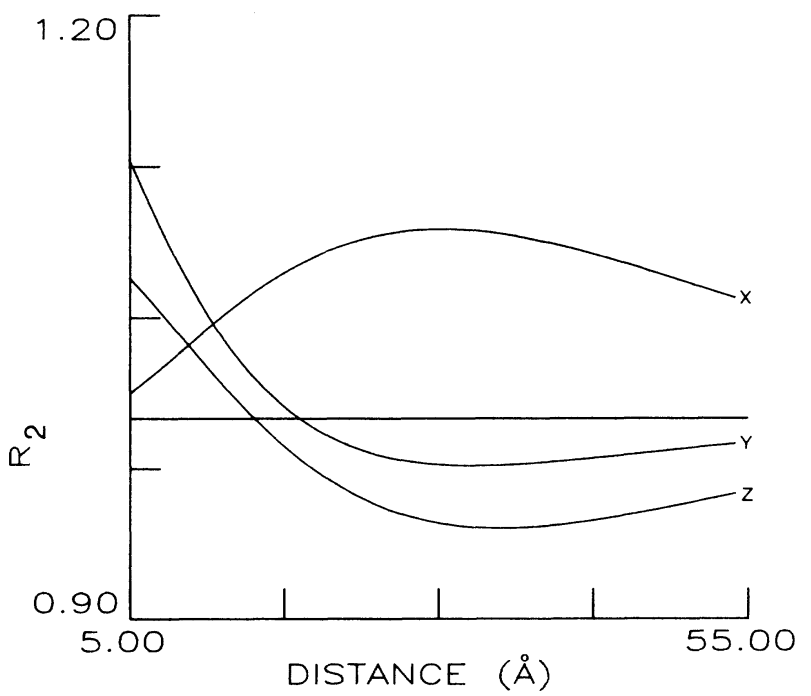

FIG. 3. Variation of $R_{2}$ with the molecule-surface distance (d) close to the SP resonance with $\omega \sim 3.74 \mathrm{eV}$ for different molecular orientations. The grating parameters are the same as in Fig. 1.

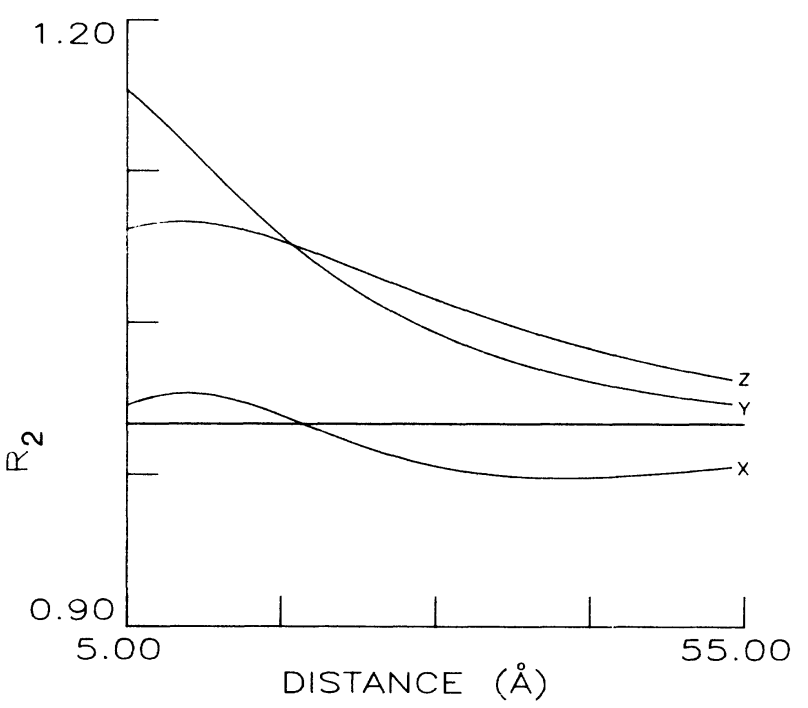

FIG. 4. Variation of $R_{2}$ with the molecule-surface distance (d) at an emitting frequency off SP resonance with $\omega=5 \mathrm{eV}$. The other parameters are the same as in Fig. 3.

Fig. 3, where now the emitting frequency is set at $\omega=5$ eV. We observe in this case that the molecules lying along the grating experience most of the diminution in the decay rate due to the surface roughness. Furthermore, we observe that all curves in Figs. 3 and 4 show the correct asymptotic behavior towards the flat-surface limit as the molecule is located further and further from the surface, as one would expect. Just for comparison of different elements, we show in Fig. 5 a similar graph as in Fig. 2 for a gold grating. We observe that the SP effect is much smaller in the gold case when compared to the silver grating. As is well known, this is due to the larger imaginary part of the dielectric constant of gold. ${ }^{25}$

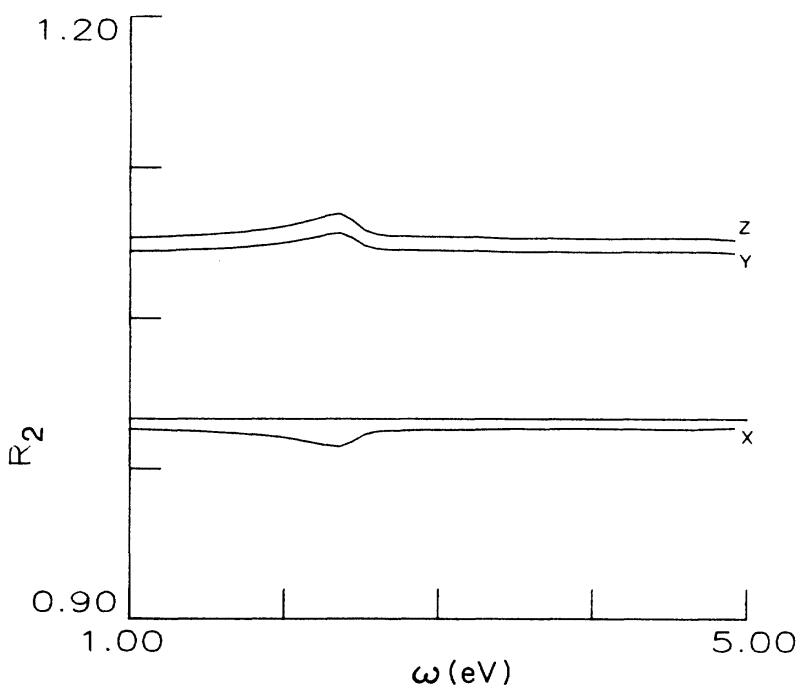

FIG. 5. The same graph as in Fig. 2, but for a gold grating surface. 


\section{DISCUSSION AND CONCLUSION}

From all the previous numerical results, we can conclude that the effects of the periodic roughness on molecular decay rates depend very much on the emitting frequency $(\omega)$, molecule-surface distance $(d)$, dielectric properties $(\epsilon)$ of the metal, and orientations of the molecules. In particular, we observe that, depending on various different conditions, the roughness can lead to either enhanced or diminished decay rates as compared to the flat-surface value. This leaves open the possibility that roughness may indeed be the origin for the discrepancies observed in the RB experiments. ${ }^{17,20}$ It is possible that under some of their experimental conditions, the molecules were oriented in such a way that most of them experience an enhanced damping, while in other situations more molecules may feel the diminution effect caused by the roughness, so that on the average, the roughness contributions become minor, if not at all disappear. It is also possible that an overall decrease in the decay rate from the flat-surface value can be observed in an experiment of the RB type. Moreover, the SP effect has been observed to be minor for the case of gold, which might explain to a certain extent why the RB experimental results for gold agree well with the flat-surface CPS theory. Furthermore, the results obtained in this work may find interesting applications since, as we have mentioned in the Introduction, roughness of the periodic type can be controlled by various grooving and deposition techniques. ${ }^{26,27}$ In this regard, we would like to mention one example of the photoabsorption-dissociation processes. $^{28-30}$ As is well known, in contrast to ordinary SERS, the enhanced SP field does not necessarily lead to enhancement of such processes due to the fact that the surface line-broadening effect here serves as a competing mechanism to suppress the process. However, as indicated above, if we can arrange the process to occur on a grating surface ${ }^{29}$ with the parameters $\omega, d, \epsilon$ and molecular orientations properly adjusted so that diminution of the decay rate will result because of the surface roughness, then this undesired linebroadening effect will be suppressed to a great extent, and hence a more efficient surface-enhanced photoabsorptiondissociation process will be achieved. Since the control of all these parameters is indeed feasible in practice (e.g., $d$ can be controlled by the "fatty acid" or the "three-layer sandwich matrix" technique and the molecular orientation by properly polarizing the driving laser field ${ }^{17}$ ), we feel that it would be worthwhile for experimentalists to pursue in the future the practical side of such surfaceenhanced processes, as proposed here by taking advantage of the regularity of the roughness of the surface.

\section{ACKNOWLEDGMENTS}

This research was supported by the Office of Naval Research, the Air Force Office of Scientific Research (AFSC), United States Air Force, under Contract No. F49620-86-C-0009, and the National Science Foundation under Grant No. CHE-8620274.
'See, e.g., H. Kuhn, J. Chem. Phys. 53, 101 (1970); V. Celli, A. Marvin, and F. Toigo, Phys. Rev. B 11, 1779 (1975); R. R. Chance, A. Prock, and R. Silbey, J. Chem. Phys. 62, 2245 (1975).

${ }^{2}$ M. Fleischmann, P. J. Handra, and A. J. McQuillan, Chem. Phys. Lett. 26, 163 (1974).

${ }^{3}$ See, e.g., M. Moskovits, Rev. Mod. Phys. 57, 783 (1985).

${ }^{4}$ A. A. Maradudin, in Surface Polaritons, edited by V. M. Agranovich and D. L. Mills (North-Holland, Amsterdam, 1982), Chap. 10.

${ }^{5}$ See, e.g., S. S. Jha, J. R. Kirtley, and J. C. Tsang, Phys. Rev. B 22, 3973 (1980).

${ }^{6}$ See, e.g., M. Meier, A. Wokaun, and P. F. Liao, J. Opt. Soc. Am. B 2, 931 (1985); M. P. Cline, P. W. Burber, and R. K. Chang, ibid. 3, 15 (1986).

${ }^{7}$ J. Arias, P. K. Aravind, and H. Metiu, Chem. Phys. Lett. 85, 404 (1982).

${ }^{8}$ P. J. Feibelman, Prog. Surf. Sci. 12, 287 (1982).

${ }^{9}$ See Ref. 7 and A. Adams, R. W. Rendell, W. P. West, H. P. Broida, P. K. Hansma, and H. Metiu, Phys. Rev. B 21, 5565 (1980).

${ }^{10}$ P. K. Aravind and H. Metiu, Chem. Phys. Lett. 74, 301 (1980).

${ }^{11}$ A. A. Maradudin and D. L. Mills, Phys. Rev. B 11, 1392 (1975).

${ }^{12}$ T. Rahman and A. A. Maradudin, Phys. Rev. B 21, 504 (1980).

${ }^{13}$ P. K. Aravind, E. Hood, and H. Metiu, Surf. Sci. 109, 95 (1981).
${ }^{14} \mathrm{~K}$. H. Drexhage, in Progress in Optics $X I I$, edited by E. Wolf (North-Holland, Amsterdam, 1974), p. 165ff, and references therein.

${ }^{15}$ I. Langmuir J. Am. Chem. Soc. 39, 1848 (1917); K. B. Blodgett, ibid. 57, 1007 (1935).

${ }^{16}$ R. R. Chance, A. Prock, and R. Silbey, Adv. Chem. Phys. 37, 1 (1978), and references therein.

${ }^{17}$ R. Rossetti and L. E. Brus, J. Chem. Phys. 73, 572 (1980).

${ }^{18}$ See, e.g., A. Campion, A. R. Gallo, C. B. Harris, H. J. Robota, and P. M. Whitmore, Chem. Phys. Lett. 73, 447 (1980).

${ }^{19}$ H. Metiu, in Surface-Enhanced Raman Scattering, edited by R. K. Chang and T. E. Furtak (Plenum, New York, 1981).

${ }^{20}$ R. Rossetti and L. E. Brus, J. Chem. Phys. 76, 1146 (1982).

${ }^{21}$ P. B. Johnson and R. W. Christy, Phys. Rev. B 6, 4370 (1972).

${ }^{22}$ A. Sommerfeld, Ann. Phys. (Leipzig) 28, 665 (1909); ibid. 81, 1135 (1926); and Partial Differential Equations of Physics (Academic, New York, 1949).

${ }^{23}$ T. Rahman and D. L. Mills, Phys. Rev. B 21, 1432 (1980).

${ }^{24}$ W. H. Weber and C. F. Eagen, Opt. Lett. 4, 236 (1979).

${ }^{25}$ See, e.g., J. R. Kirtley, S. S. Jha, and J. C. Tsang, Solid State Commun. 35, 509 (1980).

${ }^{26}$ For recent observation of periodic deposition, see, e.g., S. R. J. Brueck and D. J. Ehrlich, Phys. Rev. Lett. 48, 1678 (1982).

${ }^{27}$ D. A. Jelski and T. F. George, J. Appl. Phys. 61, 2353 (1987).

${ }^{28}$ A. Nitzan and L. E. Brus, J. Chem. Phys. 74, 5321 (1981).

${ }^{29}$ P. T. Leung and T. F. George, J. Chem. Phys. 85, 4729 (1986).

${ }^{30}$ P. T. Leung and T. F. George, Chem. Phys. Lett. 134, 375 (1987). 